\title{
RF GUN OPTIMIZATION STUDY *
}

\section{A. Hofler\#, P. Evtushenko, Jefferson Lab, 12000 Jefferson Ave., Newport News, VA 23606, U.S.A. M. Krasilnikov, DESY, D-15738 Zeuthen, Germany}

\section{Abstract}

Injector gun design is an iterative process where the designer optimizes a few nonlinearly interdependent beam parameters to achieve the required beam quality for a particle accelerator. Few tools exist to automate the optimization process and thoroughly explore the parameter space. The challenging beam requirements of new accelerator applications such as light sources and electron cooling devices drive the development of RF and SRF photo injectors. RF and SRF gun design is further complicated because the beam is space charge dominated. A genetic algorithm (GA) has been successfully used to optimize DC photo injector designs at Cornell University [1] and Jefferson Lab [2]. We propose studying how GA techniques can be applied to the design of RF and SRF gun injectors. In this paper, we report on the initial phase of the study where we model and optimize a system that has been benchmarked with beam measurements and simulation.

\section{RF GUN MODEL: PHOTO INJECTOR TEST FACILITY ZEUTHEN (PITZ)}

The PITZ RF gun has a straightforward and compact $\sim 6$ $\mathrm{m}$ layout [3]. This study concentrates on its front end (2 $\mathrm{m})$ : bucking solenoid, 1.5 cell $1300 \mathrm{MHz} \mathrm{RF}$ gun, and emittance compensation (main) solenoid. The distance from the gun cathode to the downstream end of the main solenoid structure is $0.34 \mathrm{~m}$. The bucking solenoid is used to zero the magnetic field at the cathode. The performance goal for this gun is to produce an electron beam with $1 \mathrm{nC}$ bunch charge and $1-2 \pi \mathrm{mm}$ mrad transverse normalized emittance [4].

Table 1: Particle distribution parameters [3]

\begin{tabular}{|c|c|}
\hline Total Charge & $1.65 \mathrm{nC}$ \\
\hline \multicolumn{2}{|c|}{ Spatial Distribution } \\
\hline $\mathrm{x}, \mathrm{y}$ & $\begin{array}{l}\text { radially symmetric } \\
\sigma=0.485 \mathrm{~mm} \mathrm{rms}\end{array}$ \\
\hline $\mathrm{Z}$ & $\begin{array}{l}\text { plateau } \\
\text { rise time } 6 \text { ps } \\
\text { width } 24 \text { ps (FWHM) }\end{array}$ \\
\hline \multicolumn{2}{|c|}{ Momentum Distribution } \\
\hline $\mathrm{x}, \mathrm{y}, \mathrm{z}$ & $\begin{array}{l}\text { isotropic } \\
\text { energy spread }=0.55 \mathrm{eV}\end{array}$ \\
\hline $\begin{array}{l}\text { Number of } \\
\text { Macroparticles }\end{array}$ & 10000 \\
\hline
\end{tabular}

The model $[3,5,6,7]$ we used for this gun reproduces

*Notice: Authored by Jefferson Science Associates, LLC under U.S. DOE Contract No. DE-AC05-06OR23177. The U.S. Government retains a non-exclusive, paid-up, irrevocable, world-wide license to publish or reproduce this manuscript for U.S. Government purposes.

"hofler@jlab.org major features of published results and is sufficient for this study. Fig. 1 is an example showing how charge transmission from a photoemission RF gun is sensitive to the phase difference between the laser and the RF, represented here by RF phase. The simulation calculates the charge $0.78 \mathrm{~m}$ from the cathode where a Faraday cup is located. The width and the general trend of the nonzero charge region are in agreement with a previously published RF phase scan [3]. The particle distribution description is in Table 1.

With this model, we perform two sets of optimizations. Both are two parameter optimizations varying RF phase and peak main solenoid field strength to minimize the transverse normalized emittance. The first is a manual parameter scan finding the minimum in a free space drift downstream of the gun. The second compares a manual parameter scan with an automated GA based search $[1,8]$ and minimizes the emittance at the end of a longer beam line.

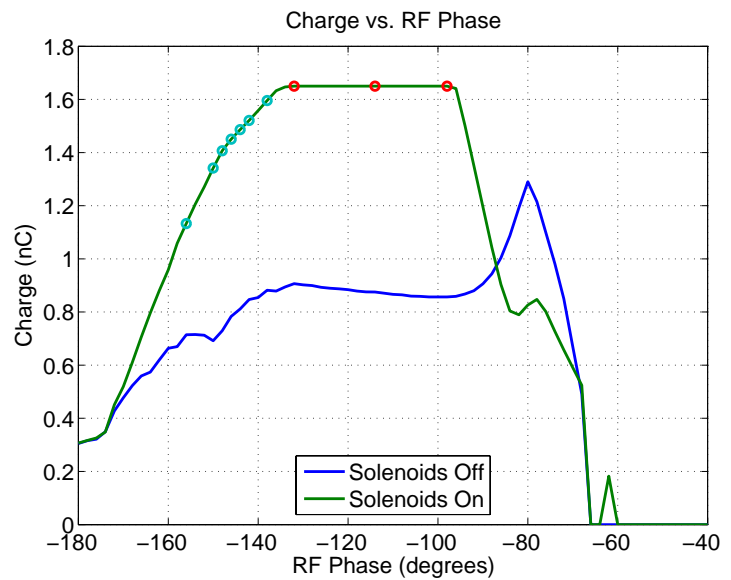

Figure 1: Charge transmission as a function of RF phase for $1.65 \mathrm{nC}$ bunch charge at the cathode. For the solenoids on case, the main solenoid is set to $300 \mathrm{~A}$ and bucking solenoid set to 21.2 A (Table 2).

Table 2: Settings for main and bucking solenoids [5]

\begin{tabular}{|c|c|c|c|}
\hline \multicolumn{2}{|c|}{ Main Solenoid } & \multicolumn{2}{c|}{ Bucking Solenoid } \\
\hline $\begin{array}{c}\text { Current } \\
\text { (A) }\end{array}$ & $\begin{array}{c}\text { Peak Field } \\
\text { Strength (T) }\end{array}$ & Current (A) & $\begin{array}{c}\text { Peak Field } \\
\text { Strength } \\
\text { (T) }\end{array}$ \\
\hline 240 & -0.141 & 16.9 & 0.00927 \\
\hline 280 & -0.165 & 19.8 & 0.0108 \\
\hline 320 & -0.188 & 22.6 & 0.0123 \\
\hline
\end{tabular}




\section{MANUAL TWO PARAMETER SCAN}

Using Fig. 1 as a guide, we manually optimize the front end with an enclosed beam path from the cathode the Faraday cup at $0.78 \mathrm{~m}$ followed by a $5.22 \mathrm{~m}$ free space drift. We use the drift space to observe the beam dynamics in the absence of beam line apertures. Both solenoids are used (Table 2).

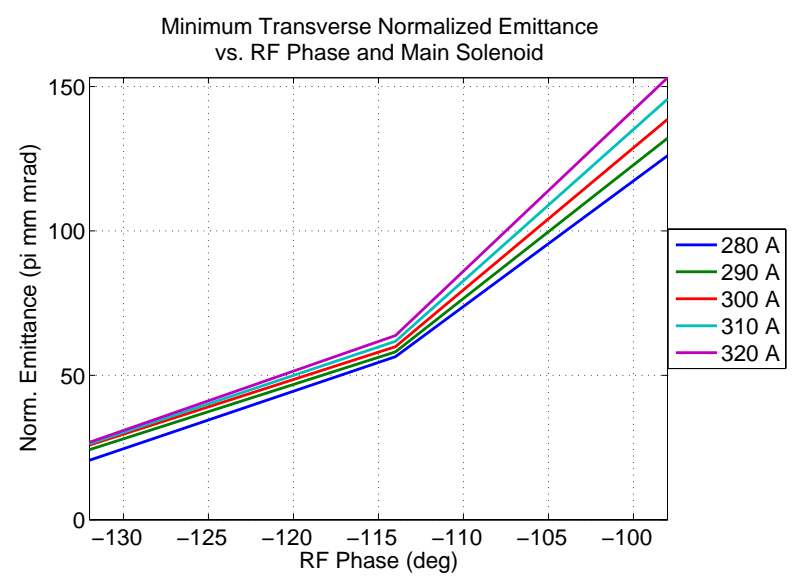

Figure 2: Minimum transverse normalized emittance in free space region for full bunch charge transmission.

In the full transmission region (red points in Fig. 1), the minimum transverse normalized emittance achieved in the free space drift is greater than $20 \pi \mathrm{mm}$ mrad and occurs for RF phase $-132^{\circ}$ and main solenoid setting $280 \mathrm{~A}$. This means that PITZ cannot meet its emittance goal with maximum transmission. This agrees with PITZ operational experience [5]. In Fig. 2, the trends are that the minimum transverse normalized emittance decreases with decreasing RF phase and solenoid setting.

For the teal points in the $\left[-156^{\circ},-138^{\circ}\right] \mathrm{RF}$ phase range in Fig. 1, simulations show that the minimum transverse normalized emittance continues to decrease with RF phase passing through a minimum at $-146^{\circ}$. There is a similar dependence on solenoid setting with the minimum occurring at $280 \mathrm{~A}$. At RF phase $-146^{\circ}$ and main solenoid setting $280 \mathrm{~A}$, the minimum emittance is $3.9 \pi \mathrm{mm}$ mrad. This is within a factor of 2-4 of the performance goal for the PITZ gun. It is notable that the maximum energy gain occurs at $-142^{\circ}$ not $-146^{\circ}$. In measurements of the PITZ gun, the minimum emittance is achieved at an RF phase that is a few degrees less than the maximum energy gain phase, and neither phase supports maximum transmission [5].

Figs. 3 and 4 are representative of the results for the RF phases in this range. These plots show how the minima for emittance and beam size move away and back toward the RF gun as the main solenoid setting increases. For the $280 \mathrm{~A}$ and $290 \mathrm{~A}$ settings, the emittance decreases slowly for 1.5-2.5 $\mathrm{m}$ beyond the Faraday cup location, and this provides the injector designer flexibility in accelerating element placement to capture the small emittance. If the solenoid setting is too weak, e.g. $240 \mathrm{~A}$ or $250 \mathrm{~A}$, the spot size increases monotonically from the exit of the gun despite having a relatively small emittance at the Faraday cup location.

Transverse Normalized Emittance vs. $\mathbf{z}$ for Phase $=-146 \mathrm{deg}$.

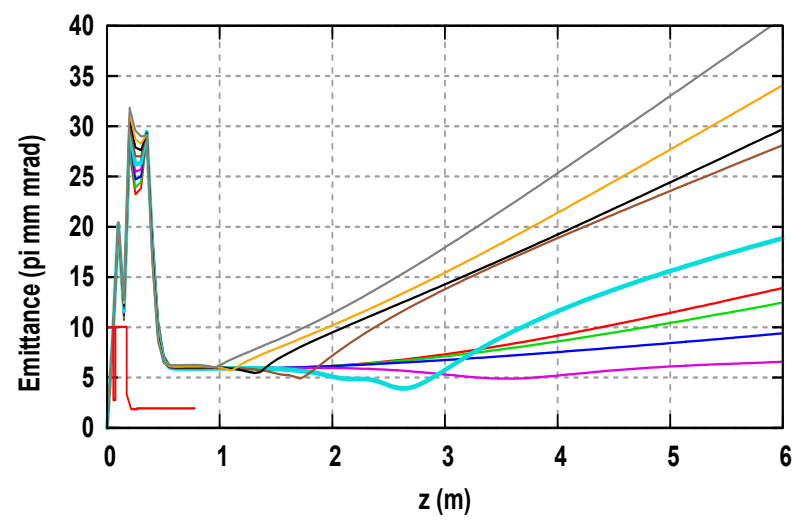

Main Sol $=240 \mathrm{~A} \square$
Main Sol $=250 \mathrm{~A} \square$
Main Sol $=260 \mathrm{~A} \square$
Main Sol $=270 \mathrm{~A} \square$
Main Sol $=280 \mathrm{~A}$

Main Sol $=290 \mathrm{~A}$ Main Sol $=300 \mathrm{~A}$

Main Sol $=310 \mathrm{~A}$

Main Sol $=320 \mathrm{~A}$

Figure 3: Transverse normalized emittance along the simulation beam line for RF phase $-146^{\circ}$ for several main solenoid settings. An arbitrarily scaled profile of the beam enclosure is provided for reference.

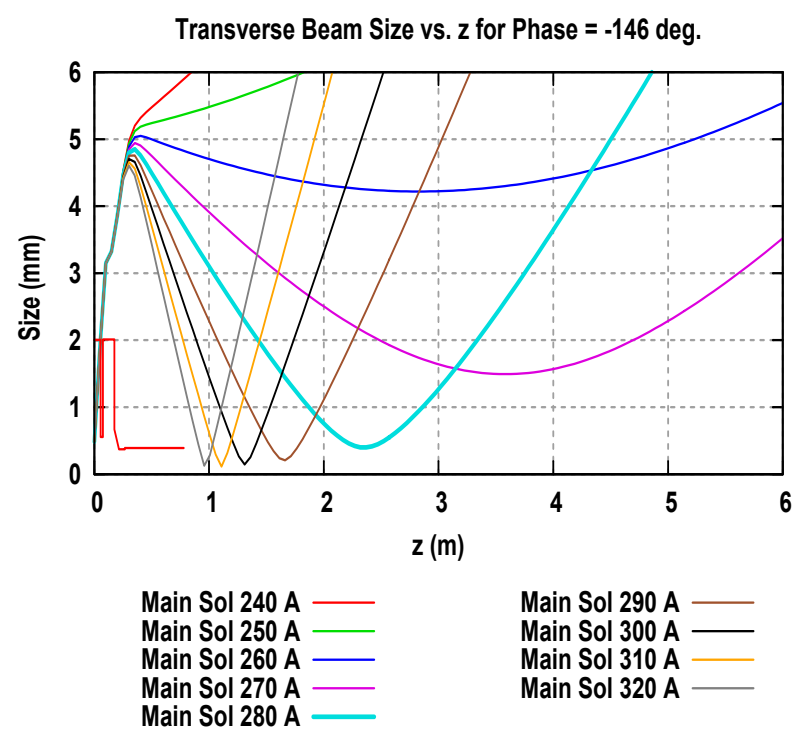

Figure 4: Transverse beam size along the simulation beam line for RF phase $-146^{\circ}$ for several main solenoid settings. An arbitrarily scaled profile of the beam enclosure is provided for reference.

\section{PARAMETER SCAN AND GA OPTIMIZATION COMPARISON}

In this optimization, the beam line extends $1.618 \mathrm{~m}$ from the cathode to a view screen. The bucking solenoid is off. For the parameter scan, the RF phase is varied from $-152^{\circ}$ to $-132^{\circ}$ in $1^{\circ}$ steps and the main solenoid is varied from $285 \mathrm{~A}$ to $305 \mathrm{~A}$ in $1 \mathrm{~A}$ steps for a total of 441 simulations. Fig. 5. shows that for the parameter scan, the minimum emittance and beam size occur when the RF 
phase is $\left[-146^{\circ},-144^{\circ}\right]$ and the main solenoid is [289 A,292 A]. For the GA optimization, the same ranges for RF Phase and main solenoid setting are the same as the parameter scan, but the GA creates a randomly distributed initial population. The GA is configured to run 16 generations of 60 individuals each for a total of 960 simulations. The optimal ranges for minimum emittance and beam size are RF phase $\left[-147^{\circ},-142.5^{\circ}\right]$ and the main solenoid [288 A,294 A]. Both methods find the same general minimum emittance operating range. They also reproduce the general form of previously published results [3]. The GA roughly converged to this region in 6 generations or 360 simulations.

$$
\text { Manual Scan }
$$

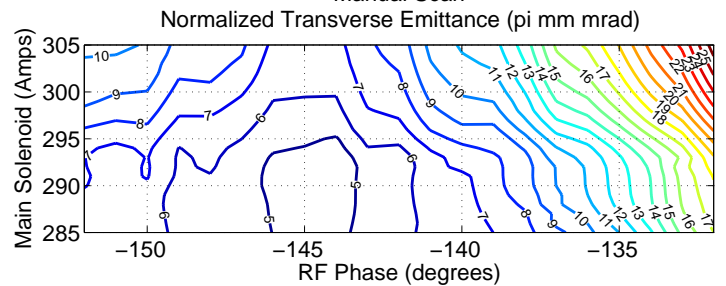

RMS Beam Size (mm)

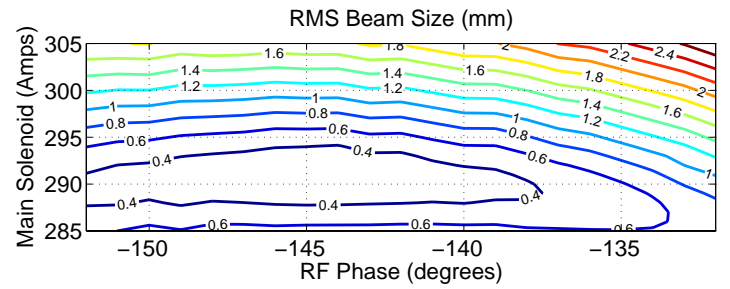

Figure 5: Parameter scan contour plots for normalized emittance and beam size.
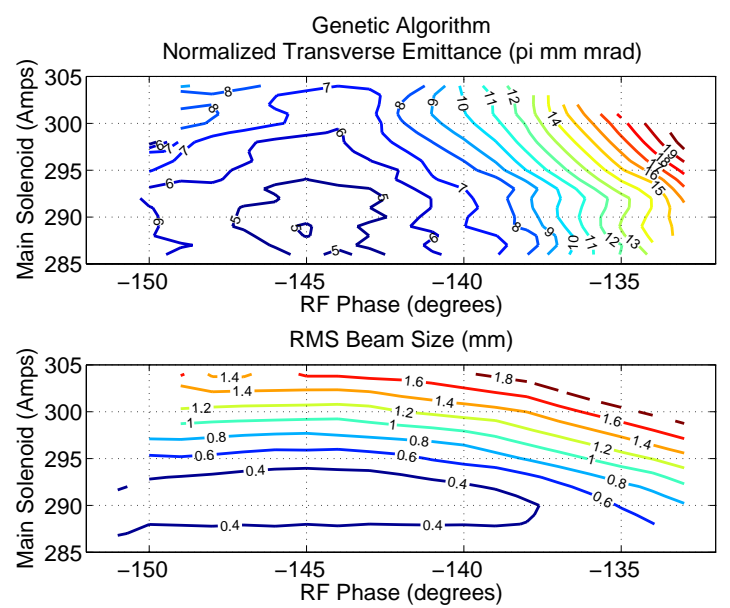

Figure 6: Emittance and beam size contour plots for GA optimization.

\section{CONCLUSION}

Injector design requires optimization of many parameters. To explore the multidimensional parameter space, an extremely large number of numerical simulations is needed, and keeping all the results of these simulations organized and presented in a simple form is a challenge. As a result the entire parameter space may not be thoroughly sampled, and the global optimum may remain unfound. GAs are tools for automating multidimensional parameter space searches. A model of the PITZ gun has been used to evaluate the possibility of using GAs for automating RF gun optimization. The next phase of this study is to design a GA framework that includes gun cell shape as a design parameter.

\section{ACKNOWLEDGEMENTS}

We would like to acknowledge Geoffrey Krafft and Eduard Pozdeyev for sharing their expertise in injector design issues with us. We would also like to thank Lia Merminga for supporting and encouraging this study.

\section{REFERENCES}

[1] I. V. Bazarov and C. K. Sinclair, "Multivariate Optimization of a High Brightness DC Gun Photoinjector," Phys. Rev. ST Accel. Beams 8, $034202(2005)$.

[2] F.E. Hannon and C. Hernandez-Garcia, "Simulation and Optimisation of a $100 \mathrm{~mA}$ DC Photoinjector," EPAC'06, Edinburgh, June 2006, pp. 3550-2, http://www.jacow.org.

[3] M. Krasilnikov, et al., "Characterization of the electron source at the photo injector test facility at DESY Zeuthen," NIM A 528, pp. 360-5 (2004).

[4] M. Krasilnikov, et al., "Optimizing the PITZ Electron Source for the VUV-FEL," EPAC'04, Lucerne Switzerland, July 2004, p. 360, http://www.jacow.org.

[5] M. Krasilnikov, "Gun Benchmark Problem (PITZ1)," http://www-

zeuthen.desy.de/ kras/PITZProblem.html/PI.TZbenc hmark.html.

[6] J.H. Billen and L.M. Young, Poisson Superfish, http://laacg.lanl.gov/laacg/services/serv_codes.phtml.

[7] K. Floettmann, ASTRA: A Space Charge Tracking Algorithm, http://www.desy.de/ mpyflo/Astra dokumentation/.

[8] S. Bleuler, et al., PISA, "PISA - A Platform and Programming Language Independent Interface for Search Algorithms," Evolutionary Multi-Criterion Optimization (EMO): Second Int'l Conf. EMO 2003, Faro, Portugal, April 2003, pp. 494-505. 